\title{
A sessile colonic polyp showing striking morphological changes within a 2-month period
}

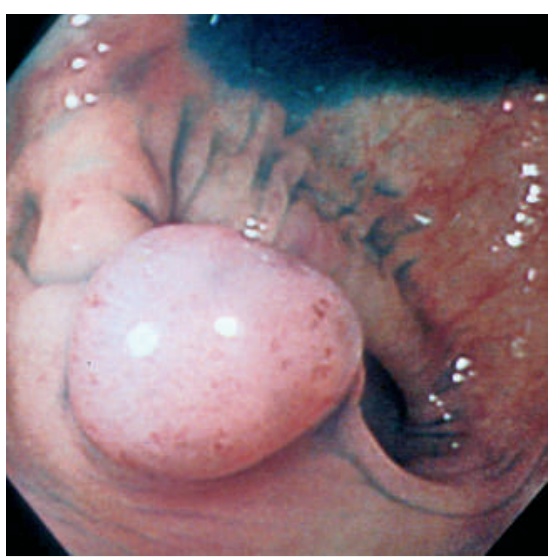

Fig. 1 The initial colonoscopy showed a sessile polyp, $10 \mathrm{~mm}$ in diameter, in the sigmoid colon.

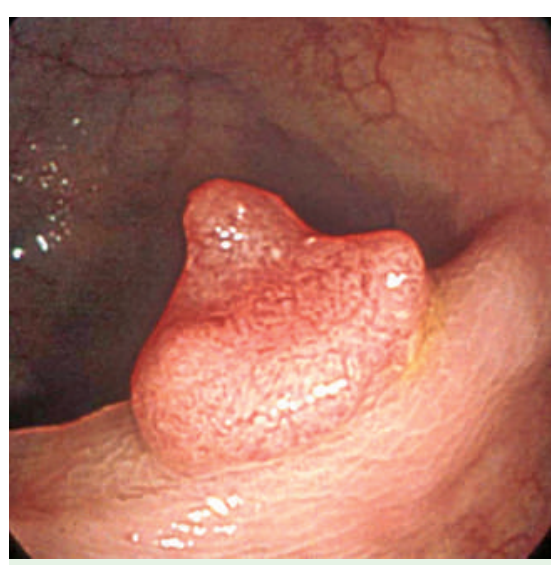

Fig. 2 At the second colonoscopy, the polyp was seen to have developed a unique "polyp on polyp" morphology.
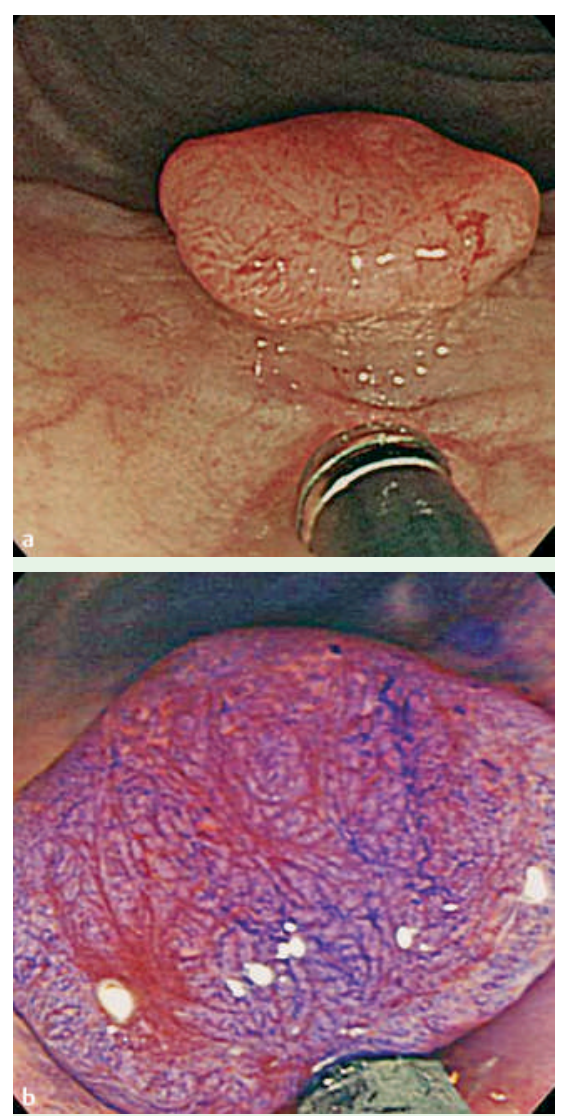

Fig. 3 a By the time of the third colonoscopy, the polyp had transformed itself into a nonpolypoid lesion, and the polyp that had developed on the original polyp's apex had disappeared. b Magnification with chromoendoscopy using $0.02 \%$ crystal violet staining showed a type $V$ pit pattern, suggesting a deeply invasive submucosal cancer.

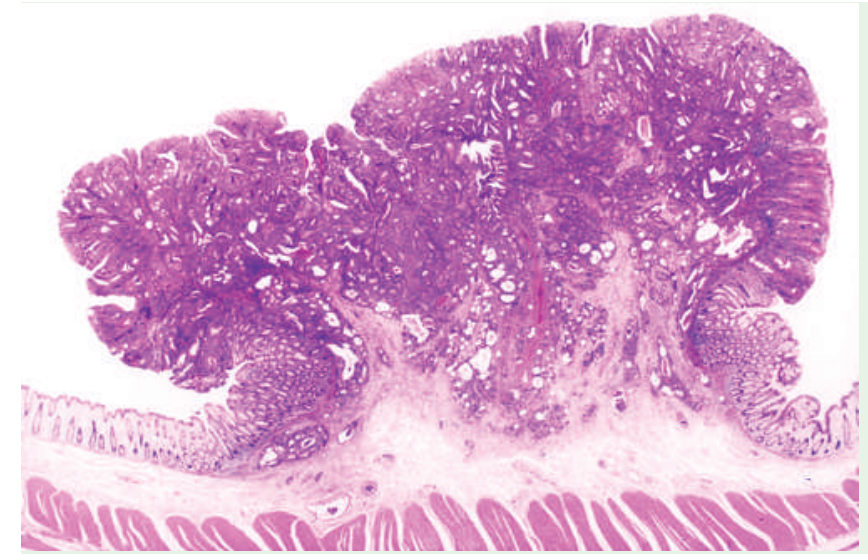

Fig. 4 Histologically, the resected specimen revealed a moderately differentiated adenocarcinoma, without an adenomatous component, that was extending deeply into the submucosal layer with vessel invasion and lymph node metastasis.
A 67-year-old man underwent total colonoscopy because he had had a positive fecal occult blood test. Colonoscopy showed a sessile polyp, $10 \mathrm{~mm}$ in diameter, in the sigmoid colon ( $\bullet$ Fig. 1). Six weeks later, he was scheduled for hospitalization to undergo endoscopic resection of this polyp. At this second colonoscopy the polyp was seen to have developed a morphologically unique "polyp on polyp" appearance ( $\bullet$ Fig. 2). Because invasive cancer was suspected on the basis of the endoscopic appearances, the endoscopic removal was discontinued and surgical resection was recommended. Nine weeks after the initial colonoscopy the patient underwent a third colonoscopy for endoscopic tattooing before surgery. Surprisingly, the polyp had transformed into a nonpolypoid lesion with a type $\mathrm{V}$ pit pattern ( Fig. 3). Laparoscopy-assisted colectomy was performed, and on histological examination the resected specimen revealed a moderately differentiated adenocarcinoma without an adenomatous component, that extended deeply into the submucosal layer with vessel invasion and lymph node metastasis (๑ Fig. 4).

The development and growth of colorectal cancers has been analyzed retrospectively using radiographic images of lesions that have been overlooked at initial examinations, but almost all of these lesions were followed up after a long interval [1-3]. This sessile polyp exhibited marked morphological change into a flat elevated early invasive cancer within an extremely short period. The mechanisms underlying this kind of morphological change are unclear. It is possible that endoscopic biopsy and submucosal injection used for diagnosis or removal of a polyp could induce such changes [4], but these procedures were not performed in this case. The growth rates of colorectal tumors are reported to accelerate when the lesions invade the submucosa [5]. Considering the morphological changes that became evident in such a short period, it is possible that this lesion was already cancerous at the outset. This rapid and marked change also indicated its biological aggressiveness and, in fact, despite its small size, it was found to have deeply invaded the submucosa, with vessel invasion and nodal involvement. Although this behavior seems to be very unusual, this type of progression should be taken into consideration if colorectal polyps are left untreated. 
Endoscopy_UCTN_Code_CCL_1AD_2AB

H. Nakamura ${ }^{1}$, K. Fu ${ }^{2}$, A. Parra-Blanco ${ }^{3}$, A. Yamamura ${ }^{4}$, Y. Oono ${ }^{5}$, Y. Iriguchi ${ }^{5}$, J. Matumoto ${ }^{6}$, Y. Kaji ${ }^{2}$, T. Ishikawa² Department of Gastroenterology, Chofu Surgical Clinic, Tokyo, Japan

2 Department of Radiology, Dokkyo Medical University, Shimotuga, Tochigi, Japan

3 Department of Gastroenterology, Hospital Universitario de Canarias, Santa Cruz de Tenerife, Tenerife, Canary Islands, Spain

4 Department of Pathology, Tokyo Metropolitan Tama Cancer Detection Center, Tokyo, Japan

${ }^{5}$ Department of Gastroenterology, Tokyo Metropolitan Tama Cancer Detection Center, Tokyo, Japan

${ }^{6}$ Department of Surgery, Tokyo Metropolitan Fuchu Hospital, Tokyo, Japan

\section{References}

1 Umetani N, Masaki T, Watanabe T et al. Retrospective radiographic analysis of nonpedunculated colorectal carcinomas with special reference to tumor doubling time and morphological change. Am J Gastroenterol 2000; 95: 1794-1799

2 Matsui T, Yao T, Iwashita A. Natural history of early colorectal cancer. World J Surg 2000; 24: $1022-1028$

3 Matsui T, Tsuda S, Iwashita A et al. Retrospective endoscopic study of developmental and configurational changes of early colorectal cancer: eight cases and a review of the literature. Dig Endosc 2004; 16: 1-8

4 Matsuda K, Masaki T, Abo Y et al. Rapid growth of residual colonic tumor after incomplete mucosal resection. J Gastroenterol 1999; 34: 260-263

5 Matsui T, Tsuda S, Yao Ket al. Natural history of early colorectal cancer: evolution of a growth curve. Dis Colon Rectum 2000; 43: S18-S22
Bibliography

DOI 10.1055/s-2007-966665

Endoscopy 2007; 39: E279-E280

(c) Georg Thieme Verlag KG Stuttgart · New York . ISSN 0013-726X

Corresponding author

\section{K. Fu, MD, PhD}

Department of Radiology

Dokkyo Medical University 880 Kitakobayashi

Mibu

Shimotuga

Tochigi 321-0293

Japan

Fax: +81-282-86-5678

fukuangi@hotmail.com 